\title{
On a Computational Advantageous Voigt Regularization for Geophysical Flows
}

\section{Igor Oliveira Monteiro ${ }^{1}$}

Programa de Pós-Graduação em Matemática Aplicada, UFRGS, Porto Alegre, RS,

Instituto Federal de Educação, Ciência e Tecnologia do Rio Grande do Sul, Rio Grande, RS

Carolina Cardoso Manica ${ }^{2}$

Instituto de Matemática, UFRGS, Porto Alegre, RS

\begin{abstract}
In this paper we study a linearized Crank-Nicolson in time and Finite Element in space algorithm for the BV-Voigt regularization model of geophysical flows, which presents interesting advantages from the computational point of view. We prove the algorithm conserves energy and is unconditionally stable and optimally convergent. Lastly, we show that the BV-Voigt model provides accurate solutions and compares favorably with a related regularization model in a coarse mesh, a case in which the BV model solution degenerates.
\end{abstract}

Keywords. Barotropic Vorticity model, Voigt regularization, geophysical flow modeling.

\section{Introduction}

Geophysical fluid dynamics studies flows influenced by Earth's rotation such as atmospheric and oceanic flows. The Barotropic Vorticity (BV) model is one of the simplest models used to simulate geophysical flows. In dimensionless form $[2,8]$, it is defined as

$$
\begin{aligned}
R o \frac{\partial \omega}{\partial t}+R o J(\psi, \omega)-\frac{\partial \psi}{\partial x}-\left(\frac{\delta_{M}}{L}\right)^{3} \Delta \omega & =F \text { in } \Omega \times(0, T) \\
\Delta \psi & =-\omega \text { in } \Omega \times(0, T) \\
\omega(x, 0) & =\omega_{0}(x) \text { in } \Omega
\end{aligned}
$$

with $\omega$ representing vorticity, $\psi$ stream function, Jacobian $J(\cdot, \cdot)$, Munk scale $\delta_{M}$, length scale $L$, Rossby number $R o$ and forcing term $F$.

Despite of being one of the simplest model for geophysical flows, its numerical simulation is still computationally challenging when long-time integration is required, as in the climate modeling case [8], for instance. Traditionally, essentially dissipative techniques such as eddy viscosity parametrization have been used to model the under-resolved part of

\footnotetext{
${ }^{1}$ igoromonteiro@gmail.com, igor.monteiro@riogrande.ifrs.edu.br

${ }^{2}$ carolina.manica@ufrgs.br
} 
the flow. However, increasing artificial viscosity tends to reduce variability and nonlinear structures can be destroyed by excessive dissipation $[1,2]$.

Our goal in this paper is to study a Voigt regularization of the BV model, which has significant computational advantages compared to other regularizations such as BV- $\alpha[6]$ and BV-Bardina models [7]. BV-Voigt is a regularization of the BV model based on the Navier-Stokes-Voigt (NSV) equations [5], an incompressible viscoelastic model which is a smooth regularization of Navier-Stokes equations [4]. In [5] it is showed that NSV reduces the stiffness of direct numerical simulations of turbulent flows, with small impact in the energy containing scales. Unlike the BV- $\alpha$ and BV-Bardina models, in the BVVoigt there is no need to solve a filter equation, reducing by one the number of equations in the model. Moreover, we considered a linearized Crank-Nicolson in time and Finite Element (FE) in space algorithm for BV-Voigt based on an adaptation of the Baker's method presented in [3] which avoids the point fixed iteration that would be necessary to solve the resulting nonlinear system from the original Crank-Nicolson method. With these two techniques we obtained a computational efficient, energy conserving, unconditionally stable and optimally convergent algorithm in order to apply the BV model in geophysical simulations.

The paper is organized as follows: Section 2 presents the FE scheme and in Section 3 the algorithm is analysed with respect to stability and convergence. In Section 4, we present simulations in which we estimate convergence rates and evaluate the BV-Voigt solutions in coarse meshes. Finally, concluding remarks are summarized in Section 5.

\section{The finite element scheme and preliminaries}

Let $\Omega \subset \mathbb{R}^{2}$ be a polygonal domain and $\tau_{h}$ be a regular discretization of $\Omega$. Let $H^{1}$ be the Sobolev space $W_{2}^{1}(\Omega)$ and $H_{0}^{1}$ its subspace with zero boundary condition. Let $Y_{h}$ be the continuous finite element (FE) with $k$ th degree polynomial on each element of the triangulation $\tau_{h}$ and $X_{h}$ be the subspace of $Y_{h}$ with zero boundary values. Denote by $\langle\cdot, \cdot\rangle$ and $\|\cdot\|$ the inner product and norm in $L^{2}(\Omega)$ and $\|\cdot\|_{k}$ for the norm in the space $H^{k}$.

The following lemma is helpful in the subsequent analysis (see [6]).

Lemma 2.1 (Skew-symmetry of the trilinear form). Given $\psi, \xi, \chi \in X_{h}$,

$$
\langle J(\psi, \chi), \xi\rangle=-\langle J(\psi, \xi), \chi\rangle
$$

Our motivation for studying the BV-Voigt model is the search for efficient, unconditionally stable and accurate methods in order to simulate geophysical flows. Below we propose a FE in space and Crank-Nicolson in time discretization. As in [8] we considered slip boundary conditions for the velocity which translate into homogeneous Dirichlet condition $\left.\omega\right|_{\partial \Omega}=0$ and the impermeability condition $\left.\psi\right|_{\partial \Omega}=0$.

Algorithm 2.1 (Crank-Nicolson - BV-Voigt model). Let $\omega_{h}^{0}$ and $\psi_{h}^{0}$ be $L^{2}(\Omega)$ projections into $X_{h}$ of $\omega_{0} \in H_{0}^{1}$ and $\psi_{0} \in H_{0}^{1}$, endtime $T, F \in L^{\infty}\left(0, T ; L^{2}(\Omega)\right)$, and timestep $\Delta t>0$. 
Set $M=\frac{T}{\Delta t}$ and for $n=0, \ldots, M-1$, find $\left(\omega^{n}, \psi^{n}\right) \in X_{h} \times X_{h}$ satisfying:

$$
\begin{aligned}
\alpha^{2} R o\left\langle\nabla \frac{\left(\omega_{h}^{n+1}-\omega_{h}^{n}\right)}{\Delta t}, \nabla \lambda\right\rangle+R o\left\langle\frac{\omega_{h}^{n+1}-\omega_{h}^{n}}{\Delta t}, \lambda\right\rangle & +R o\left\langle J\left(\psi_{h}^{n+\frac{1}{2}}, \xi\left(\omega_{h}^{n+\frac{1}{2}}\right)\right), \lambda\right\rangle \\
-\left\langle\frac{\partial \psi_{h}^{n+\frac{1}{2}}}{\partial x}, \lambda\right\rangle+\left(\frac{\delta_{M}}{L}\right)^{3}\left\langle\nabla \omega_{h}^{n+\frac{1}{2}}, \nabla \lambda\right\rangle & =\left\langle F^{n+\frac{1}{2}}, \lambda\right\rangle \forall \lambda \in X_{h}, \\
\left\langle\nabla \psi_{h}^{n+1}, \nabla \chi\right\rangle & =\left\langle\omega_{h}^{n+1}, \chi\right\rangle \forall \chi \in X_{h} .
\end{aligned}
$$

where $\alpha \geq 0$ is a given length scale, $\xi\left(\omega_{h}^{n+\frac{1}{2}}\right)=2 \omega_{h}^{n-\frac{1}{2}}-\omega_{h}^{n-\frac{3}{2}}$ and $v^{n+\frac{1}{2}}:=\frac{v^{n}+v^{n+1}}{2}$.

\section{Analysis of the scheme}

The first step in the analysis of Algorithm 2.1 is to demonstrate that it conserves a modified form of kinetic energy. We start by defining a Modified Kinetic Energy and a Energy Dissipation in the BV-Voigt model respectively by

$$
\begin{aligned}
E_{\alpha}(\psi, \omega) & :=\frac{1}{2}\|\nabla \psi\|^{2}+\frac{1}{2} \alpha^{2}\|\omega\|^{2}, \\
\epsilon(\omega) & :=\left(\delta_{M} / L\right)^{3}\|\omega\|^{2} .
\end{aligned}
$$

Then, we obtain the following lemma:

Lemma 3.1 (Conservation of kinetic energy). The solution of (1a) satisfies

$$
E_{\alpha}\left(\psi_{h}^{M}, \omega_{h}^{M}\right)+\frac{\Delta t}{R o} \sum_{n=0}^{M-1} \epsilon\left(\omega_{h}^{n+\frac{1}{2}}\right)=E_{\alpha}\left(\psi_{h}^{0}, \omega_{h}^{0}\right)+\frac{\Delta t}{R o} \sum_{n=0}^{M-1}\left\langle F^{n+\frac{1}{2}}, \psi_{h}^{n+\frac{1}{2}}\right\rangle .
$$

In particular, if $\delta_{M}=0$ and $F=0$ we have $E_{\alpha}\left(\psi_{h}^{M}, \omega_{h}^{M}\right)=E_{\alpha}\left(\psi_{h}^{0}, \omega_{h}^{0}\right)$.

Proof. Choosing $\lambda=\psi_{h}^{n+\frac{1}{2}}$ in (1a), using lemma 2.1, using $\left\langle\frac{\partial \psi_{h}^{n+\frac{1}{2}}}{\partial x}, \psi_{h}^{n+\frac{1}{2}}\right\rangle=0$ because $\psi_{h}^{n+\frac{1}{2}} \in X_{h}$ and multiplying by $\frac{\Delta t}{R o}$

$$
\begin{aligned}
& \alpha^{2}\left\langle\nabla\left(\omega_{h}^{n+1}-\omega_{h}^{n}\right), \nabla \psi_{h}^{n+\frac{1}{2}}\right\rangle+\left\langle\omega_{h}^{n+1}-\omega_{h}^{n}, \psi_{h}^{n+\frac{1}{2}}\right\rangle \\
& \quad+\frac{\Delta t}{R o}\left(\frac{\delta_{M}}{L}\right)^{3}\left\langle\nabla \omega_{h}^{n+\frac{1}{2}}, \nabla \psi_{h}^{n+\frac{1}{2}}\right\rangle=\frac{\Delta t}{R o}\left\langle F^{n+\frac{1}{2}}, \psi_{h}^{n+\frac{1}{2}}\right\rangle .
\end{aligned}
$$

Now, we have to rewrite the three terms on the LHS. For the first, subtracting (1b) in time step $n$ from (1b) in time step $n+1$ and choosing $\chi=\psi_{h}^{n+\frac{1}{2}}$ we obtain

$$
\left\langle\omega_{h}^{n+1}-\omega_{h}^{n}, \psi_{h}^{n+\frac{1}{2}}\right\rangle=\left\langle\nabla \psi_{h}^{n+1}-\nabla \psi_{h}^{n}, \nabla \psi_{h}^{n+\frac{1}{2}}\right\rangle=\frac{\left\|\nabla \psi_{h}^{n+1}\right\|^{2}-\left\|\nabla \psi_{h}^{n}\right\|^{2}}{2} .
$$

For the second term in (2), averaging in (1b) and choosing $\chi=\omega_{h}^{n+1}-\omega_{h}^{n}$,

$$
\left\langle\nabla \psi_{h}^{n+\frac{1}{2}}, \nabla \omega_{h}^{n+1}-\nabla \omega_{h}^{n}\right\rangle=\left\langle\omega_{h}^{n+\frac{1}{2}}, \omega_{h}^{n+1}-\omega_{h}^{n}\right\rangle=\frac{\left\|\omega_{h}^{n+1}\right\|^{2}-\left\|\omega_{h}^{n}\right\|^{2}}{2} .
$$


Using (3), (4) and the definition of $E_{\alpha}(\psi, \omega)$, we have

$$
E_{\alpha}\left(\psi_{h}^{n+1}, \omega^{n+1}\right)-E_{\alpha}\left(\psi_{h}^{n}, \omega^{n}\right)=\alpha^{2}\left\langle\nabla\left(\omega_{h}^{n+1}-\omega_{h}^{n}\right), \nabla \psi_{h}^{n+\frac{1}{2}}\right\rangle+\left\langle\omega_{h}^{n+1}-\omega_{h}^{n}, \psi_{h}^{n+\frac{1}{2}}\right\rangle .
$$

For the third term in (2), averaging in (1b) and choosing $\chi=\omega_{h}^{n+\frac{1}{2}}$

$$
\left\langle\nabla \psi_{h}^{n+\frac{1}{2}}, \nabla \omega_{h}^{n+\frac{1}{2}}\right\rangle=\left\|\omega_{h}^{n+\frac{1}{2}}\right\|^{2} .
$$

Using (5) and (6) in (2)

$$
E_{\alpha}\left(\psi_{h}^{n+1}, \omega^{n+1}\right)-E_{\alpha}\left(\psi_{h}^{n}, \omega^{n}\right)+\frac{\Delta t}{R o}\left(\frac{\delta_{M}}{L}\right)^{3}\left\|\omega_{h}^{n+\frac{1}{2}}\right\|^{2}=\frac{\Delta t}{R o}\left\langle F^{n+\frac{1}{2}}, \psi_{h}^{n+\frac{1}{2}}\right\rangle,
$$

from which the result follows by summing from $n=0,1, \ldots, M-1$.

Lemma 3.2 (Stability). Algorithm 2.1 is unconditionally stable. Its solutions satisfy

$$
\alpha^{2}\left\|\omega_{h}^{M}\right\|^{2}+\left\|\nabla \psi_{h}^{M}\right\|^{2}+\frac{\Delta t}{R o}\left(\frac{\delta_{M}}{L}\right)^{3} \sum_{n=0}^{M}\left\|\omega_{h}^{n+\frac{1}{2}}\right\|^{2} \leq\left(\frac{L}{\delta_{M}}\right)^{3} \frac{C^{4}}{R o} \Delta t \sum_{n=0}^{M}\left\|F^{n+\frac{1}{2}}\right\|^{2} .
$$

Proof. Now, using Cauchy-Schwarz and Young's inequalities with $\epsilon=\left(\delta_{M} / L\right)^{3}$ in $(7)$

$$
\alpha^{2} \frac{\left\|\omega_{h}^{n+1}\right\|^{2}-\left\|\omega_{h}^{n}\right\|^{2}}{2}+\frac{\left\|\nabla \psi_{h}^{n+1}\right\|^{2}-\left\|\nabla \psi_{h}^{n}\right\|^{2}}{2}+\frac{1}{2} \frac{\Delta t}{R o}\left(\frac{\delta_{M}}{L}\right)^{3}\left\|\omega_{h}^{n+\frac{1}{2}}\right\|^{2} \leq \frac{1}{2}\left(\frac{L}{\delta_{M}}\right)^{3} \frac{C^{4}}{R o} \Delta t\left\|F^{n+\frac{1}{2}}\right\|^{2},
$$

where we used $\left\|\nabla \psi_{h}^{n+\frac{1}{2}}\right\| \leq C\left\|\omega_{h}^{n+\frac{1}{2}}\right\|$ which results averaging (1b), choosing $\chi=\psi_{h}^{n+\frac{1}{2}}$ and using the Poincaré inequality. Result follows summing from $n=0,1, \ldots, M-1$.

Remark 3.1. We remark that Algorithm 2.1 is linear and $X_{h}$ is finite dimensional. Thus, for a given time $t^{n}\left(t^{n}:=n \Delta t\right)$ and following the proof of lemma 3.2, we can prove uniqueness of solutions from which existence follows as well for the entire scheme.

Theorem 3.1. Let $(\omega(t), \psi(t))$ be a smooth strong solution of the $B V$ model and $\|f\|_{\infty, k}:=$ $\operatorname{ess} \sup _{0 \leq n \leq M}\left\|f^{n}\right\|_{k}$. Suppose $\left(\omega_{h}, \psi_{h}\right) \in X_{h} \times X_{h}$ solves the approximation (1a)-(1b). Then, for $\Delta t$ small enough (in order to apply the discrete Gronwall inequality), we have

$$
R o\left\|\omega-\omega_{h}\right\|_{\infty, 0}+\left(\left(\frac{\delta_{M}}{L}\right)^{3} \sum_{n=0}^{M-1} \Delta t\left\|\nabla\left(\omega\left(t^{n+\frac{1}{2}}\right)-\omega_{h}^{n+\frac{1}{2}}\right)\right\|^{2}\right)^{1 / 2} \leq O\left(h^{k}+\Delta t^{2}+\alpha^{2}\right) .
$$

Moreover, using the result above, we have

$$
\left\|\psi-\psi_{h}\right\|_{\infty, 1} \leq O\left(h^{k}+\Delta t^{2}+\alpha^{2}\right) .
$$

Proof. Using the proof of Theorem 1 presented in [7] with $\alpha=0$, it remains only to bound the extrapolation error caused by the linearization of the nonlinear term, which can be easily bound using Taylor series and the following terms

$$
\begin{aligned}
\alpha^{2}\left\langle\nabla\left(\omega^{n+1}-\omega^{n}\right), \nabla e_{h}^{n+\frac{1}{2}}\right\rangle & \leq \frac{\epsilon}{2} \alpha^{4}\left\|\nabla\left(\omega^{n+1}-\omega^{n}\right)\right\|^{2}+\frac{1}{2 \epsilon}\left\|\nabla e_{h}^{n+\frac{1}{2}}\right\|^{2}, \\
\alpha^{2}\left\langle\nabla\left(e_{\perp}^{n+1}-e_{\perp}^{n}\right), \nabla e_{h}^{n+\frac{1}{2}}\right\rangle & \leq \frac{\epsilon}{2} \alpha^{4}\left\|\nabla\left(e_{\perp}^{n+1}-e_{\perp}^{n}\right)\right\|^{2}+\frac{1}{2 \epsilon}\left\|\nabla e_{h}^{n+\frac{1}{2}}\right\|^{2},
\end{aligned}
$$

which we have used the Cauchy-Schwarz and Young's inequalities. Moreover, $e_{h}:=\omega_{h}-$ $P \omega$ and $e_{\perp}:=\omega-P \omega$, where $P$ is the $L^{2}$ projection in $X_{h}$ (see [7]). 


\section{Numerical experiments}

Now, we present two numerical tests in order to evaluate our BV-Voigt scheme. The proposed algorithm was implemented in the software FreeFem $++^{3}$ (see [7] for details on the implementation). Table 1 presents the convergence rates estimated using the analytical solution $\psi=\exp \left[-\frac{2 \pi^{2}}{R o}\left(\frac{\delta_{M}}{L}\right)^{3}\right] \sin (\pi x) \sin (\pi y)$ (see [7]). In this table, the estimated convergence rates corroborate the theoretical convergence rates described in Theorem 3.1.

Table 1: Convergence rates for BV model with $\frac{\delta_{M}}{L}=0.02$ and $R o=1.0$.

\begin{tabular}{c|c|c|c|c|c|c|c}
\hline Element & $h^{-1}$ & $\left\|w-w_{h}\right\|_{2,1}$ & Rate & $\left\|w-w_{h}\right\|_{\infty, 0}$ & Rate & $\left\|\psi-\psi_{h}\right\|_{\infty, 1}$ & Rate \\
\hline$P_{1}$ & 4 & $5.7650 \mathrm{E}+1$ & & $1.4446 \mathrm{E}+1$ & & $3.2264 \mathrm{E}+0$ & \\
$(\Delta t=\sqrt{h})$ & 8 & $1.6554 \mathrm{E}+1$ & 1.80 & $3.8776 \mathrm{E}+0$ & 1.90 & $1.0477 \mathrm{E}+0$ & 1.62 \\
& 16 & $5.5979 \mathrm{E}+0$ & 1.56 & $9.8756 \mathrm{E}-1$ & 1.97 & $3.7818 \mathrm{E}-1$ & 1.47 \\
& 32 & $2.3353 \mathrm{E}+0$ & 1.26 & $2.4806 \mathrm{E}-1$ & 1.99 & $1.6378 \mathrm{E}-1$ & 1.21 \\
& 64 & $1.1005 \mathrm{E}+0$ & 1.09 & $6.2089 \mathrm{E}-2$ & 2.00 & $7.8322 \mathrm{E}-2$ & 1.06 \\
\hline$P_{2}$ & 4 & $5.4320 \mathrm{E}+1$ & & $1.7167 \mathrm{E}+1$ & & $3.8608 \mathrm{E}+0$ & \\
$(\Delta t=h)$ & 8 & $1.3669 \mathrm{E}+1$ & 1.99 & $4.3071 \mathrm{E}+0$ & 1.99 & $9.6949 \mathrm{E}-1$ & 1.99 \\
& 16 & $3.4491 \mathrm{E}+0$ & 1.99 & $1.0779 \mathrm{E}+0$ & 2.00 & $2.4250 \mathrm{E}-1$ & 2.00 \\
& 32 & $8.7346 \mathrm{E}-1$ & 1.98 & $2.6957 \mathrm{E}-1$ & 2.00 & $6.0631 \mathrm{E}-2$ & 2.00 \\
& 64 & $2.2214 \mathrm{E}-1$ & 1.98 & $6.7339 \mathrm{E}-2$ & 2.00 & $1.5158 \mathrm{E}-2$ & 2.00
\end{tabular}

In the second test we evaluate the BV-Voigt model in the traditional Double Gyre Wind Forcing benchmark for $R o=0.0016$ and $\left(\delta_{M} / L\right)^{3}=0.02$ (for more details, see [7]). In this experiment, the solution of BV model is very sensitive to the mesh resolution. In Figures 1(a) and 2(a) we present, respectively, the stream function and vorticity high resolution solution obtained in a uniform triangular mesh with 16,384 triangles $(33,153$ degrees of freedom, corresponding to a grid with $64 \times 128$ squares).

Finally, we evaluate the solutions obtained in a coarse uniform triangular mesh with 1,024 triangles (2,145 degrees of freedom, corresponding to a grid with $16 \times 32$ squares). Figures 1(b) and 2(b) present, respectively, the stream function and vorticity solutions obtained with the BV model. In this case, the stream function solution intensifies when compared to the high resolution solution, causing significant discrepancy also in the vorticity field. After that, we test three techniques to fix the mesh resolution problem: increasing $\delta_{M} / L$ in the BV model (artificial viscosity technique), the BV- $\alpha$ model and the BV-Voigt model. Figures 1(c) and 2(c)) show that solution degenerates using the artificial viscosity technique $\left(\delta_{M} / L=0.04\right)$. On the other hand, the BV-Voigt (Figures $1(\mathrm{~d})$ and $2(\mathrm{~d})$ ) and the BV- $\alpha$ (Figures 1(e) and 2(e)) are able to reproduce the high resolution solution. Moreover, we observe that the BV-Voigt model compares favorably with the BV- $\alpha$ model (see the min and max stream function values presented in Figure 1).

\footnotetext{
${ }^{3}$ www.freefem.org
} 

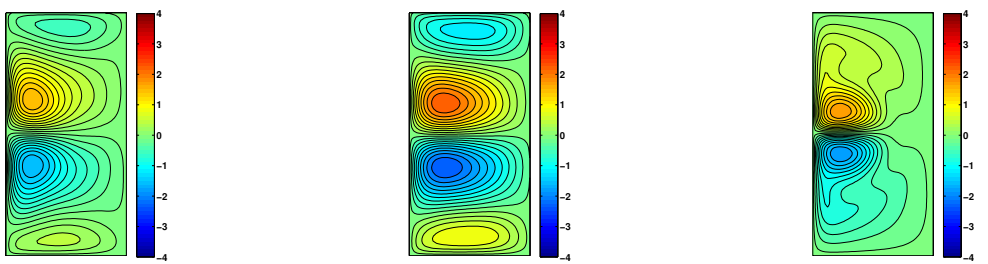
(a) BV (high res.), $\quad \psi_{\text {min }}=-1.39$,
(b) BV (Coarse), $\psi_{\text {min }}=-2.281$, $\psi_{\max }=1.39$ $\psi_{\max }=2.361$
(c) $\mathrm{BV}+$ Art. Visc. (Coarse), $\quad \psi_{\min }=-$ 1.794, $\psi_{\max }=1.828$
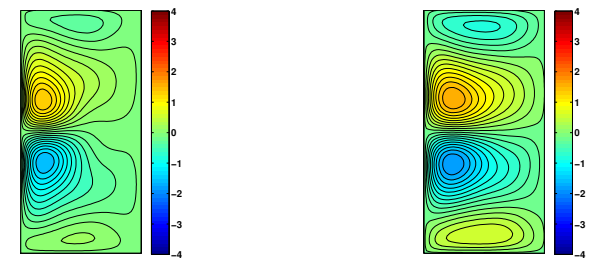

$$
\begin{aligned}
& \text { (d) } \quad \text { BV-Voigt } \\
& \text { (Coarse), } \quad \psi_{\min }=- \\
& 1.513, \psi_{\max }=1.513
\end{aligned}
$$

(e) BV- $\alpha$ (Coarse), $\psi_{\min }=-1.829$,

$\psi_{\max }=1.745$

Figure 1: Mean fields of stream function for the high resolution BV model (a) and for the coarse BV (b), BV + artificial viscosity (c), BV-Voigt (d) and BV- $\alpha$ (e) models.

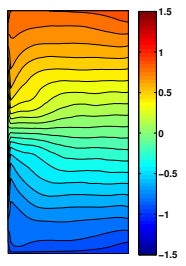

(a) Vort. - BV mod. high res.

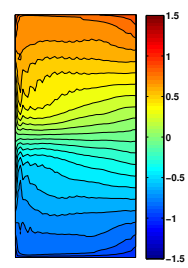

(b) Vort. - BV mod. Coarse

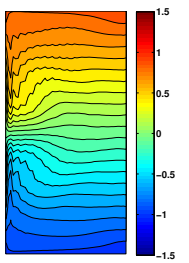

(d) Vort. - BV-Voigt mod. - Coarse

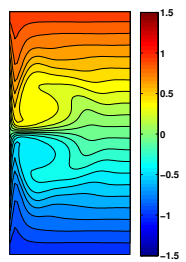

(c) Vort. - BV mod. Coarse + Art. Visc.

Figure 2: Mean vorticity fields for various models.

\section{Concluding remarks}

In this paper we proposed a Crank-Nicolson in time and FE in space algorithm for BVVoigt model of geophysical flows, which presents good advantages from the computational point of view. We proved the algorithm conserves energy, is unconditionally stable and 
optimally convergent. Lastly, we tested the BV-Voigt model in a traditional benchmark test which we showed it produces an accurate solution in a coarse mesh, the same in which the BV model solution degenerates. Also, in this test, the BV-Voigt model compared favorably with the analogous BV- $\alpha$ model.

\section{References}

[1] R. Greatbatch and B. Nadiga, Four-Gyre Circulation in a Barotropic Model with Double-Gyre Wind Forcing, Journal of Phys. Ocean., vol. 30, 1461-1471, (2000), DOI: $10.1175 / 1520-0485(2000) 030\langle 1461$ FGCIAB $\rangle 2.0 . C O ; 2$.

[2] D. Holm and B. Nadiga, Modeling Mesoscale Turbulence in the Barotropic DoubleGyre Circulation, Journal of Phys. Ocean., vol. 33, 2355-2365, (2003), DOI: 10.1175/1520-0485(2003)033〈2355:MMTITB $\rangle 2.0 . \mathrm{CO} ; 2$.

[3] R. Ingram, A new linearly extrapolated Crank-Nicolson time-stepping scheme for the Navier-Stokes equations, Math. of Comp., vol. 82, 1953-1973, (2013), DOI: 10.1090/s0025-5718-2013-02678-6.

[4] V. Kalantarov, B. Levant and E. Titi, Gevrey Regularity for the Attractor of the 3D Navier-Stokes-Voight Equations, J. Nonlinear Sci., vol. 19, 133-152, (2008), DOI: 10.1007/s00332-008-9029-7.

[5] B. Levant, F. Ramos and E. Titi, On the statistical properties of the 3D incompressible Navier-Stokes-Voigt model, Comm. in Math. Sci., vol. 8, 277-293, (2010), DOI: 10.4310/cms.2010.v8.n1.a14.

[6] I. Monteiro and C. Manica, Improving numerical accuracy in a regularized barotropic vorticity model of geophysical flow, vol. 5, 317-338, Int. Journal of Num. Model. Ser. B, (2014).

[7] I. Monteiro, C. Manica and L. Rebholz, Numerical study of a regularized barotropic vorticity model of geophysical flow, Num. Met. Partial Eq., (2015), DOI: 10.1002/num.21956.

[8] O. San, A. Staples, Z. Wand and T. Iliescu, Approximate Deconvolution Large Eddy Simulation of a Barotropic Ocean Circulation Model, Oc. Model., vol. 40, 120-132, (2011), DOI: 10.1016/j.ocemod.2011.08.003. 